\title{
Anchored phylogenomics illuminates the skipper butterfly tree of life
}

\author{
Emmanuel F. A. Toussaint ${ }^{1 *}$ D, Jesse W. Breinholt ${ }^{1,2}$, Chandra Earl ${ }^{1}$, Andrew D. Warren ${ }^{1}$, Andrew V. Z. Brower ${ }^{3}$, \\ Masaya Yago ${ }^{4}$, Kelly M. Dexter ${ }^{1}$, Marianne Espeland ${ }^{5}$, Naomi E. Pierce ${ }^{6}$, David J. Lohman ${ }^{7,8,9}$ and Akito Y. Kawahara ${ }^{1}$
}

\begin{abstract}
Background: Butterflies (Papilionoidea) are perhaps the most charismatic insect lineage, yet phylogenetic relationships among them remain incompletely studied and controversial. This is especially true for skippers (Hesperiidae), one of the most species-rich and poorly studied butterfly families.

Methods: To infer a robust phylogenomic hypothesis for Hesperiidae, we sequenced nearly 400 loci using Anchored Hybrid Enrichment and sampled all tribes and more than 120 genera of skippers. Molecular datasets were analyzed using maximum-likelihood, parsimony and coalescent multi-species phylogenetic methods.
\end{abstract}

Results: All analyses converged on a novel, robust phylogenetic hypothesis for skippers. Different optimality criteria and methodologies recovered almost identical phylogenetic trees with strong nodal support at nearly all nodes and all taxonomic levels. Our results support Coeliadinae as the sister group to the remaining skippers, the monotypic Euschemoninae as the sister group to all other subfamilies but Coeliadinae, and the monophyly of Eudaminae plus Pyrginae. Within Pyrginae, Celaenorrhinini and Tagiadini are sister groups, the Neotropical firetips, Pyrrhopygini, are sister to all other tribes but Celaenorrhinini and Tagiadini. Achlyodini is recovered as the sister group to Carcharodini, and Erynnini as sister group to Pyrgini. Within the grass skippers (Hesperiinae), there is strong support for the monophyly of Aeromachini plus remaining Hesperiinae. The giant skippers (Agathymus and Megathymus) once classified as a subfamily, are recovered as monophyletic with strong support, but are deeply nested within Hesperiinae.

Conclusions: Anchored Hybrid Enrichment sequencing resulted in a large amount of data that built the foundation for a new, robust evolutionary tree of skippers. The newly inferred phylogenetic tree resolves long-standing systematic issues and changes our understanding of the skipper tree of life. These resultsenhance understanding of the evolution of one of the most species-rich butterfly families.

Keywords: Anchored hybrid enrichment, Butterfly phylogenomics, Coalescent multi-species, Hesperiidae, Lepidoptera, Maximum likelihood, Molecular systematics, Papilionoidea, Parsimony, Target capture

\section{Background}

DNA sequencing and molecular phylogenetics have provided powerful tools to reconstruct the tree-of-life (ToL). Using a small number of gene fragments sampled across the genome, phylogenetic relationships of diverse groups have been inferred and their taxonomy revised. However, it has become evident that sampling such a small fraction of the genome often does not contain

\footnotetext{
*Correspondence: Emmanuel.touss1@gmail.com

${ }^{1}$ Florida Museum of Natural History, University of Florida, Gainesville, Florida 32611, USA

Full list of author information is available at the end of the article
}

sufficient phylogenetic information to resolve long-standing systematic conundrums, or to allow inference of robust hypotheses of relationship for higher taxonomic groups. In the past decade, next-generation sequencing methods have stimulated the evolution of phylogenetics into phylogenomics, providing a means to generate data from hundreds or thousands of loci across the genome at relatively low cost. Target capture sequencing coupled with next-generation sequencing is a recently developed method for sampling large numbers of loci throughout the genome $[1,2]$. Anchored Hybrid Enrichment (AHE [3]) is a form of target capture that

(C) The Author(s). 2018 Open Access This article is distributed under the terms of the Creative Commons Attribution 4.0 International License (http://creativecommons.org/licenses/by/4.0/), which permits unrestricted use, distribution, and 
captures moderately conserved loci by designing probes with dense tiling across loci in several lineages and enables the enrichment of orthologous loci from distantly related taxa. Genomic regions that hybridize with probes are enriched with PCR and can then be sequenced. AHE can work well for degraded DNA from older specimens [4], permitting the use of specimens with DNA too fragmented for PCR or reduced representation methods requiring restriction enzymatic cleavage. The use of AHE in systematics is still in its infancy, but pioneering studies have shown the astonishing potential of this method to fully resolve deeper parts of the ToL.

Trees constructed with AHE have clarified relationships among taxa that diverged long ago. Prum et al. [5] used AHE to sequence 259 loci and infer a novel and robust time-tree for birds, highlighting the power of the approach to shed light on ancient avian divergences that could not be resolved with 19 loci [6]. At a finer scale, Tucker et al. [7] resolved a long-standing debate regarding the classification of teiid lizards using AHE. Stout et al. [8] used AHE to infer the deeper-level phylogenetics of freshwater carps and loaches (Cypriniformes), while previous studies using a few loci inferred incongruent phylogenetic trees. Similarly, deep relationships across the jumping spider (Salticidae) tree-of-life were resolved using AHE [9]. Relationships within the angiosperm ToL that remained uncertain for decades have been clarified with AHE (e.g., sages [10]; sugarbushes [11]). A few studies have used AHE to address fine-scale evolutionary patterns within derived clades of the ToL.

Studies employing AHE have also resolved relationships among recently diverged lineages. Domingos et al. [12] used AHE to tackle species delimitation of Tropidurus lizards. Similarly, Ruane et al. [13] demonstrated that species trees estimated with a handful of loci were potentially biased and that an AHE approach allowed collection of sufficient sequence data to provide a robust phylogenetic estimate of pseudoxyrhophiine snakes. Anchored Hybrid Enrichment has also been shown useful for resolving different taxonomic levels across the spider ToL [14]. Early studies using AHE sampled relatively few taxa. Therefore, the utility of AHE to resolve both ancient and recent divergences in a single clade of the ToL has not been examined thoroughly [15].

With more than a million described species, insects are the most species-rich animal group on Earth, but only a few studies have examined their evolution using datasets including hundreds of loci sampled broadly across the genome. Young et al. [16] inferred a robust phylogeny of flower flies (Syrphidae) with AHE, and the nodal support of their tree was stronger than previous studies. Similarly, Winterton et al. [17] reconstructed a dated phylogeny of lacewings with an unprecedented level of resolution and robust nodal support. Haddad et al. [18] shed light on deep relationships of longhorn beetles (Cerambycidae) using an AHE dataset comprising 522 loci, providing the first robust phylogenetic hypothesis for the family. Using the same beetle AHE probe kit, Shin et al. [19] provided a much clearer picture of weevil phylogenetic relationships and evolutionary history. Breinholt et al. [20] used several AHE datasets to examine relationships across Lepidoptera and tested the utility of AHE to resolve relationships within superfamilies. This study corroborated the placement of butterflies as a derived moth clade, and demonstrated that strong nodal support can be achieved with AHE data. Recently, Espeland et al. [21] inferred a new phylogenomic framework for butterflies at the tribal level using an AHE approach, resolving the placement of clades that had been controversial for decades. The present study delves more deeply and examines relationships among butterflies at the genus-level.

Hesperiidae (or "skippers") is a family of butterflies comprising more than 4200 described species [22], with highest diversity in the Neotropics. These fast-flying insects feed on angiosperms as larvae, are occasionally crepuscular, and are immediately recognized by their hook-shaped antennae, relatively chunky bodies, and characteristic silhouette while at rest. The family is cosmopolitan, and representatives can be found from the Arctic to the tropics, at nearly all elevations and in all terrestrial habitat types [23]. Unlike species in other butterfly families whose adults are frequently large and charismatic, skippers are often small and overlooked. Nevertheless, studies of their distribution, ecology and behavior have led to important discoveries. Skippers play a central role in pollination ecology. Recent studies have shown that species with long proboscides feed significantly more often on flowers with long nectar spurs [24] and are non-pollinating nectar thieves [25], affecting the pollination success of flowers with purloined nectar. However, in some angiosperm lineages such as orchids, skippers appear to be important pollinators [26]. Hesperiids have also been used as a model to study foraging strategies, demonstrating that switches between potential flower species for nectar occur in response to plant population density rather than specializing on particular flower species [27]. Competition for flower access between Neotropical hummingbirds and skippers has been suggested as one of the first examples of vertical partitioning of food resources in the context of interference competition [28]. Skippers have also been used as models in conservation and pest control pilot studies. A study of the silver-spotted skipper, Hesperia comma, in Britain showed that butterfly population decline could be arrested through adequate landscape-scale conservation 
policies coupled with favorable climatic change [29, 30]. During the end of the twentieth century, the banana skipper, Erionota thrax, had become a major pest across Southeast Asia and the Pacific and has been the subject of early pest management studies in Hawaii and New Guinea [31]. Skippers host plant use shows interesting ecological patterns that can be studied in an evolutionary context. For instance, Sahoo et al. [32] suggested that the evolution of monocot feeding in some lineages was associated with higher diversification rates. Dating of divergence times in butterflies suggests that skippers originated ca. 80 million years ago in the late Cretaceous $([21,32,33])$.

Hesperiidae was historically thought to be the sister group to the remaining butterfly families, and has been treated as a suborder [34], a superfamily [35] or a family [36]. A century passed before molecular phylogenetic analyses seemed to substantiate skippers' sister-relationship to all other butterflies (e.g., [37]). However, strong evidence now suggests that skippers are the sister-group to the "butterfly-moths", Hedylidae, and are nested within the Papilionoidea [20, 21, 38-40].

The first relatively comprehensive phylogenetic analyses of Hesperiidae were conducted only a decade ago by Warren et al. [23, 41]. Those results, which were based on three loci and morphological data for 196 genera, supported the monophyly of the family, and were used to revise subfamily and tribal boundaries. Sahoo et al. [32, 42] recently increased taxon and locus sampling to include 10 gene regions and approximately 300 species but, like Warren's studies, did not recover strong support for most subfamilial and tribal-level relationships within the family (Fig. 1). For example, the placement of the monotypic Australian regent skipper Euschemon rafflesia (Euschemoninae) remained poorly supported (but see [43]). Similarly, the placement of the subfamily Eudaminae changed depending on the dataset and analysis employed [32, 42]. Finally, as found by Warren et al. [23, 41], the monophyly of Pyrginae and of some tribes within Hesperiinae was uncertain, with low nodal support for the placement of the different tribes (Fig. 1). These studies reveal the limits of phylogenetic inference using a small number of markers to infer higher-level relationships in rapidly diversifying taxa. A sufficiently large number of markers might help provide stronger support for the tree, but obtaining dozens or hundreds of homologous loci is impractical and expensive using traditional Sanger sequencing. Here, we implement a phylogenomic approach using AHE of hundreds of protein-coding loci to improve resolution of the skipper ToL and provide the first robust hypothesis for subfamilial and tribal relationships, on which patterns of diversification and biogeographic questions can be addressed.

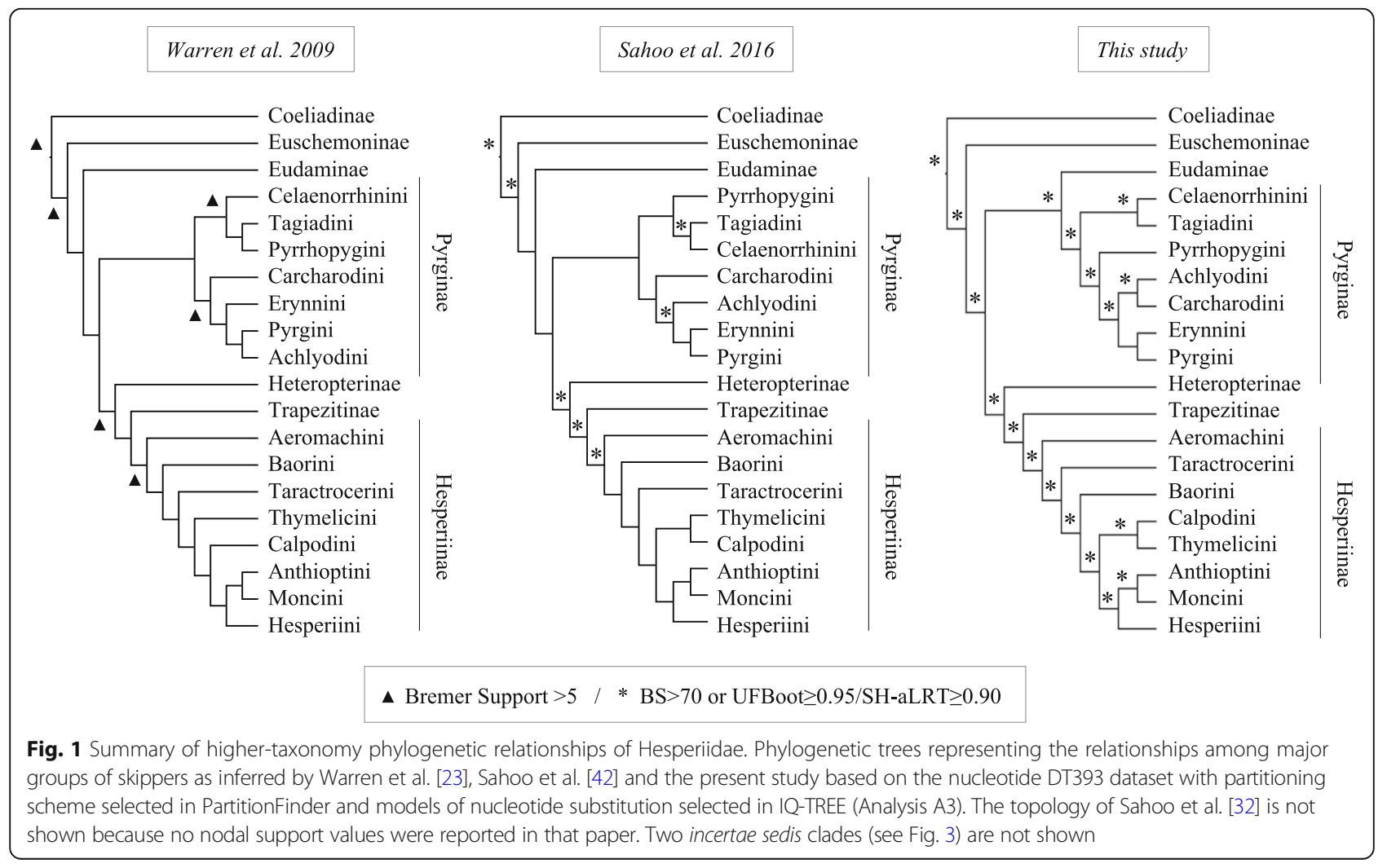




\section{Methods}

\section{Taxon sampling}

We sampled 130 species from all recognized hesperiid subfamilies and tribes. Multiple exemplars from each tribe and genus were included for particularly species-rich lineages (Additional file 1: Table S1). We included three species of Hedylidae and one representative from each of the five other recognized butterfly families as outgroups (Additional file 1: Table S1). Specimens were collected from the wild and stored as (1) dried specimens in envelopes, (2) bodies preserved entirely in $>95 \% \mathrm{EtOH}$, or (3) legs kept in vial containing $>95 \%$ EtOH. For each specimen, the right-wing pair was preserved as a voucher, following a published protocol [44]. All extracts are stored at $-80{ }^{\circ} \mathrm{C}$ at the McGuire Center for Lepidoptera and Biodiversity (MGCL), Florida Museum of Natural History (Gainesville, USA).

\section{DNA extraction and Illumina sequencing}

Genomic DNA was extracted using Omni Prep Genomic Extraction kits (G-Biosciences, Saint Louis, USA), which includes a chloroform phase separation step. Frozen, fresh or dried Lepidoptera tissue from the thorax or leg was homogenized in a 1:100 dilution of proteinase $\mathrm{K}$ to genomic lysis buffer in a centrifuge at $1200 \mathrm{rpm}$ for $2.5 \mathrm{~min}$ at $25{ }^{\circ} \mathrm{C}$. Samples were incubated overnight at $56{ }^{\circ} \mathrm{C}$ for approximately $12-18 \mathrm{~h}$. The plate was then centrifuged at $3000 \mathrm{rpm}$ for $3 \mathrm{~min}$ before $200 \mu \mathrm{L}$ of chloroform was added to each sample. Samples were then centrifuged for $10 \mathrm{~min}$ at $4000 \mathrm{rpm}$ to complete phase separation. Up to $80 \%$ of the supernatant was transferred to new, sterile $2.0 \mathrm{~mL}$ microtiter tubes and treated with $50 \mu \mathrm{L}$ of DNA stripping buffer. Samples were incubated at $56{ }^{\circ} \mathrm{C}$ for $10 \mathrm{~min}$, and tubes were cooled to room temperature before being centrifuged at $3000 \mathrm{rpm}$ for $3 \mathrm{~min}$ before $100 \mu \mathrm{L}$ of precipitation buffer was added to each sample along with $5 \mu \mathrm{L}$ of mussel glycogen $(10 \mathrm{mg} / \mathrm{ml})$. Samples were mixed and centrifuged at $6000 \mathrm{rpm}$ for $20 \mathrm{~min}$ to form a pellet. The supernatant was transferred to sterile $1.5 \mathrm{~mL}$ tubes and $500 \mu \mathrm{L}$ of 2-propanol was added to each. Solutions were gently mixed and incubated at $-20 \mathrm{C}$ for approximately $30 \mathrm{~min}$ before being centrifuged at $4000 \mathrm{rpm}$ for $10 \mathrm{~min}$. The DNA was cleaned by adding $700 \mu \mathrm{L}$ ethanol and centrifuging at $4000 \mathrm{rpm}$ for $10 \mathrm{~min}$. Subsequently, the ethanol was removed, and DNA was eluted in $50 \mu \mathrm{L}-100 \mu \mathrm{L}$ of $\mathrm{TE}$ buffer with the addition of $0.5 \mu \mathrm{L}$ of RNase solution to remove traces of RNA. Samples were incubated overnight at $4{ }^{\circ} \mathrm{C}$ before DNA quantification. Some DNA extracts from the work of Warren et al. [41] were also included for AHE sequencing (see [41] for details of their DNA extraction protocol). DNA extracts were sent to RAPiD Genomics (Gainesville, FL, USA) for library preparation, hybrid enrichment, and sequencing. Libraries were constructed by mechanical shearing of DNA to an average size of 300 bp. Following shearing, end-repair reaction and ligation of an adenine residue to the 3 '-end of the blunt-end fragments were performed to allow the ligation of barcoded adapters and PCR-amplification of the library. For AHE, a custom set of SureSelect probes (Agilent Technologies: Santa Clara, CA) were used for solution-based target enrichment of a pool containing 16 indexed libraries following the SureSelect Target Enrichment System for Illumina Paired-End Multiplexed Sequencing Library protocol. The Agilent Custom SureSelect kit used in this study (BUTTERFLY1.1 kit) is a modification (reduction from 425 to 391 loci with the best capture success across Papilionoidea and the addition of more reference sequences to improve capture) of the Papilionoidea-specific kit BUTTERFLY1.0 [21] which was derived by modifying and expanding the LEP1 probe set for Lepidoptera [20]. The BUTTERFLY1.1 kit loci names are consistent with loci names in BUTTERFLY1.0. This new kit includes 348 protein-coding loci that overlap with the AHE kit of Breinholt et al. [20].

\section{Data assembly}

The data assembly pipeline largely followed Breinholt et al. [20]. TrimGalore! 0.4.0 (https://www.bioinformatics. babraham.ac.uk/projects/trim_galore/) was used to filter raw sequences with a minimum size of $30 \mathrm{bp}$. The assembly of each locus was performed using iterative baited assembly, with a Python script used to execute USEARCH [45] and Bridger [46] to assemble loci iteratively for each taxon. Orthology of sequences was assessed by using the single-hit and genome mapping location criteria defined by Breinholt et al. [20]. Single-hit and genome mapping location criteria uses NCBI BLASTN [47], with the genomic sequence of Danaus plexippus [48] as a reference, and scripts from Breinholt et al. [20] were applied to remove sequences for which the second-best hit had a bit score $>90 \%$ of the best hit bit score and confirming the location of the best hit to the orthologous loci. Contamination checks were conducted with USEARCH and Python scripts, to identify and remove nearly identical sequences across different subfamilies and genera. The final cleaned, assembled sequences trimmed to the probe region were aligned using MAFFT v. 7.245 [49] and strict consensus sequences for assembled isoforms were generated using FASconCAT-G 1.02 [50].

\section{Dataset construction}

Individual gene fragment alignments were inspected and analyzed to remove stop codons, indels, sequencing errors and to assign correct protein-coding frames in AliView 1.18 [51]. To consider the potential impact of missing data, two datasets were generated: (1) a dataset comprising all 
loci that had been successfully captured, sequenced, and assembled from more than one taxon (total of 393 loci including two loci from BUTTERFLY1.0 that assembled although the probes were not included in BUTTERFLY1.1; this dataset is hereafter referred to as DT393); and (2) a more conservative dataset with fewer missing data, comprising all loci that had been successfully captured, sequenced and assembled for $\geq 70$ taxa for a total of 369 loci, hereafter referred to as DT369. The two datasets were separately concatenated using FASconCAT-G 1.02. The raw individual locus alignments, cleaned concatenated alignments, and partitioning files used for phylogenetic inference in this study (see below) are available on Dryad (https://doi.org/10.5061/dryad.bq10 q98).

\section{Phylogenomic analyses}

We inferred phylogenetic relationships under the maximum likelihood criterion using IQ-TREE 1.4.2 [52], in both unpartitioned and partitioned frameworks, to investigate the robustness of our inferences based on different partitioning schemes. We analyzed the dataset in three different ways: (A1) using a single concatenated alignment, (A2) using one partition per locus, and (A3) using a partitioning scheme determined in PartitionFinder 2.1.1 [53]. For the unpartitioned analyses A1 and the partitioned analyses (A2) (Table 1), the best models of nucleotide substitution were determined with the Bayesian Information criterion (BIC) using the command "TESTNEW" in IQ-TREE 1.4.2. For the partitioned analyses (A3), the optimal partitioning schemes were selected using the BIC in PartitionFinder 2.1.1. The search was based on a priori partitioning per locus with the rcluster algorithm [53] and the rcluster percentage set to 10 (default setting). Nucleotide substitution models for each partition were selected using BIC in IQ-TREE 1.4.2, across all available models including the FreeRate model $(+\mathrm{R},[54])$, which relaxes the assumption of gamma distributed rates. To assess nodal support, we performed 1000 ultrafast bootstrap replicates (UFBoot, [55]) with the "-bb" command. We also performed a SH-aLRT test [56] with 1000 replicates using the command "-alrt". The UFBoot has been shown to be largely unbiased compared to standard or alternative bootstrap strategies, and SH-aLRT has been shown to be as conservative as standard bootstrap [55]. Only nodes with support values of UFBoot $\geq 95$ and SH-aLRT $\geq 90$ were considered robust.

We also inferred species trees under the multi-species coalescent model in ASTRAL-II 4.10.0 [57]. Gene trees of each locus were estimated in IQ-TREE 1.4.2 for all 393 loci. Individual loci were not partitioned, and the best models of nucleotide substitution were selected using the BIC in IQ-TREE 1.4.2 across all available models. The maximum likelihood trees for each locus were assembled in two files, one for the 393 locus dataset and one for the 369 locus set and analyzed in ASTRAL-II 4.10.0 (Analyses A4). We performed 100 bootstrap replicates (ASTRALBoot) to assess the robustness of nodes in the final topologies, and calculated quartet scores (the number of gene tree quartets satisfied by the species tree) and normalized quartet scores (proportion of input gene tree quartet trees satisfied by the species tree). The higher the normalized quartet score, the less discordant individual gene trees are when compared to the species tree inferred in ASTRAL-II.

IQ-TREE and ASTRAL-II analyses were also conducted after translating the DT369 and DT393 nucleotide datasets into amino acids with FASconCAT-G 1.02. Unpartitioned analyses (A5) and analyses partitioned based on locus position (A6) (Table 1), were conducted

Table 1 Results of the IQ-TREE maximum likelihood phylogenetic analyses

\begin{tabular}{|c|c|c|c|c|c|}
\hline Analysis & Dataset & Data Type & Partitioning scheme & N. Partitions & Consensus LnL \\
\hline$A 1$ & DT369 & Nucleotides & None & 1 & -5115300.064 \\
\hline$A 2$ & DT369 & Nucleotides & Locus & 366 & -5080057.834 \\
\hline$A 3^{\mathrm{a}}$ & DT369 & Nucleotides & $\mathrm{PF}$ & 88 & -5077543.916 \\
\hline A5 & DT369 & Amino Acids & None & 1 & -630708.956 \\
\hline A6 & DT369 & Amino Acids & Locus & 366 & -624687.178 \\
\hline A7 & DT369 & Amino Acids & $\mathrm{PF}$ & 27 & -627253.336 \\
\hline A1 & DT393 & Nucleotides & None & 1 & -5241440.350 \\
\hline$A 2$ & DT393 & Nucleotides & Locus & 393 & -5204821.224 \\
\hline$A 3^{\mathrm{a}}$ & DT393 & Nucleotides & PF & 94 & -5203048.331 \\
\hline A5 & DT393 & Amino Acids & None & 1 & -662724.440 \\
\hline A6 & DT393 & Amino Acids & Locus & 393 & -656165.141 \\
\hline A7 & DT393 & Amino Acids & PF & 28 & -658908.662 \\
\hline
\end{tabular}

N. Partitions number of partitions, Consensus LnL log-likelihood of the consensus of all bootstrap trees, PF PartitionFinder ${ }^{a}$ the two most likely scenarios for the two datasets 
as described above for the nucleotide analyses. Due to computational limitations, the optimal partitioning schemes for the partitioned analyses using PartitionFinder 2.1.1. (A7) were selected only based on the LG model, and the models of evolution were re-estimated using IQ-TREE 1.4.2 across all available models. The ASTRAL-II analyses based on amino acids (A8) were conducted as described above for nucleotide datasets. All analyses were performed on HiPerGator 2.0, the supercomputing cluster at the University of Florida (Gainesville, USA).

The amino acid and nucleotide DT393 datasets were also analyzed under the parsimony criterion in TNT 1.5 [58] (Analyses A9 and A10). The most parsimonious trees were inferred using a heuristic approach based on traditional tree searches, treating gaps as missing data and with all characters and transformations weighted equally. Battus polydamas was used as the outgroup in all TNT analyses. TNT searches were run with 200 replications of RAS (default setting) and branch-swapping operated with tree bisection-reconnection (TBR). Bremer support (BRS) values were calculated using 500 suboptimal Wagner trees that were 5000 steps longer on which TBR branch-swapping was operated.

\section{Results and discussion}

At least 300 gene fragments were captured and sequenced from 136 of 138 species (98.6\%), with an average of 350 loci per species (Additional files 1 and 2: Tables S1 and S2). Two loci from the BUTTERFLY1.0 kit that were not included in the BUTTERFLY1.1 kit were assembled and include in the final datasets (Table 2). Two species obtained via published transcriptomes had $<300$ loci assembled; the skipper Erynnis propertius (Pyrginae, 155 loci; [59]), and the outgroup Macrosoma hedylaria, (Hedylidae, 175 loci; [40]). The capture success we obtained was remarkable, as skippers are often small, and in several cases, DNA was extracted from just two or three legs from of a single specimen. Our approach highlights the flexibility of AHE to generate genomic data for small specimens and/ or small body parts using appropriate extraction protocols and library preparation.

Results of our phylogenomic analyses are summarized in Tables 1 and 2. Partitioning resulted in an improvement of the log-likelihood in IQ-TREE analyses, regardless of the dataset used (Table 1). IQ-TREE analyses with a partitioning scheme from PartitionFinder had the best log-likelihood for both DT369 and DT393 nucleotide datasets but not for the amino acid datasets. In Fig. 2, we present the IQ-TREE topology derived from the nucleotide dataset, DT393, based on the PartitionFinder partitioning scheme (Table 1). All phylogenetic analyses recovered a highly congruent, robust tree of all major, recognized deep-level divergences in Hesperiidae (Figs. 2 and 3). The TNT analyses of the amino acid DT393 dataset recovered four most parsimonious trees of 82,982 steps, while analyses of the nucleotide DT393 dataset recovered a unique most parsimonious tree of $1,266,723$ steps. Nodal support values across the backbone were high in all analyses (see Fig. 2). The 14 backbone nodes of importance inferred using maximum likelihood had an UFBoot $\geq 95$ and SH-aLRT $\geq 90,10$ out of 14 nodes had an ASTRALBoot $\geq 95$, and 13 out of 14 nodes had a BRS $>100$ (Figs. 2 and 3). ASTRAL-II and TNT analyses recovered a few nodes with ASTRALBoot $<95$ or BRS $<5$ among the most derived nodes of the backbone, especially in the subfamily Hesperiinae. The main discrepancies among analyses are restricted to relationships within a handful of derived clades, in which branching order varies slightly, depending on the dataset and methods.

Hesperiidae is recovered as monophyletic and sister to Hedylidae with strong nodal support (UFBoot $=100$ / SH-aLRT $=100 /$ ASTRALBoot $=100 /$ BRS $>100)$ in all analyses, a result that is consistent with previous studies (e.g., [20, 38]). Within Hesperiidae, all subfamilies recognized by Warren et al. [23] are recovered as monophyletic with strong nodal support (Figs. 2 and 3). The placement of Euschemon as sister to all skippers excluding Coeliadinae was suggested by Warren et al. [23, 41] and Zhang et al. [43], but contradicted by Sahoo et al. $[32,42]$ who recovered different topologies when using different analytical methods. Here we show that Coeliadinae is the sister-group to all remaining skippers, with the monotypic Euschemoninae as sister to all skippers excluding Coeliadinae (Figs. 2 and 3).

Another significant change within the skipper ToL is the placement of the Eudaminae (Figs. 1 and 2). Many prior studies placed Eudaminae as sister to all skippers except Coeliadinae and Euschemoninae ([23, 41, 42], see Fig. 1). Here, we recover Eudaminae as sister to the spread-winged skippers of the subfamily Pyrginae (Clades I-7 in Fig. 2) with robust nodal support in all

Table 2 Results of the ASTRAL-II multispecies coalescent phylogenetic analyses

\begin{tabular}{|c|c|c|c|c|}
\hline Analysis & Dataset & Data type & Quartet score & Normalized quartet score \\
\hline A4 & DT369 & Nucleotides & 3098963077 & 0.759 \\
\hline A8 & DT369 & Amino Acids & 2035594777 & 0.501 \\
\hline A4 & DT393 & Nucleotides & 3179504945 & 0.760 \\
\hline A8 & DT393 & Amino Acids & 2082078892 & 0.501 \\
\hline
\end{tabular}




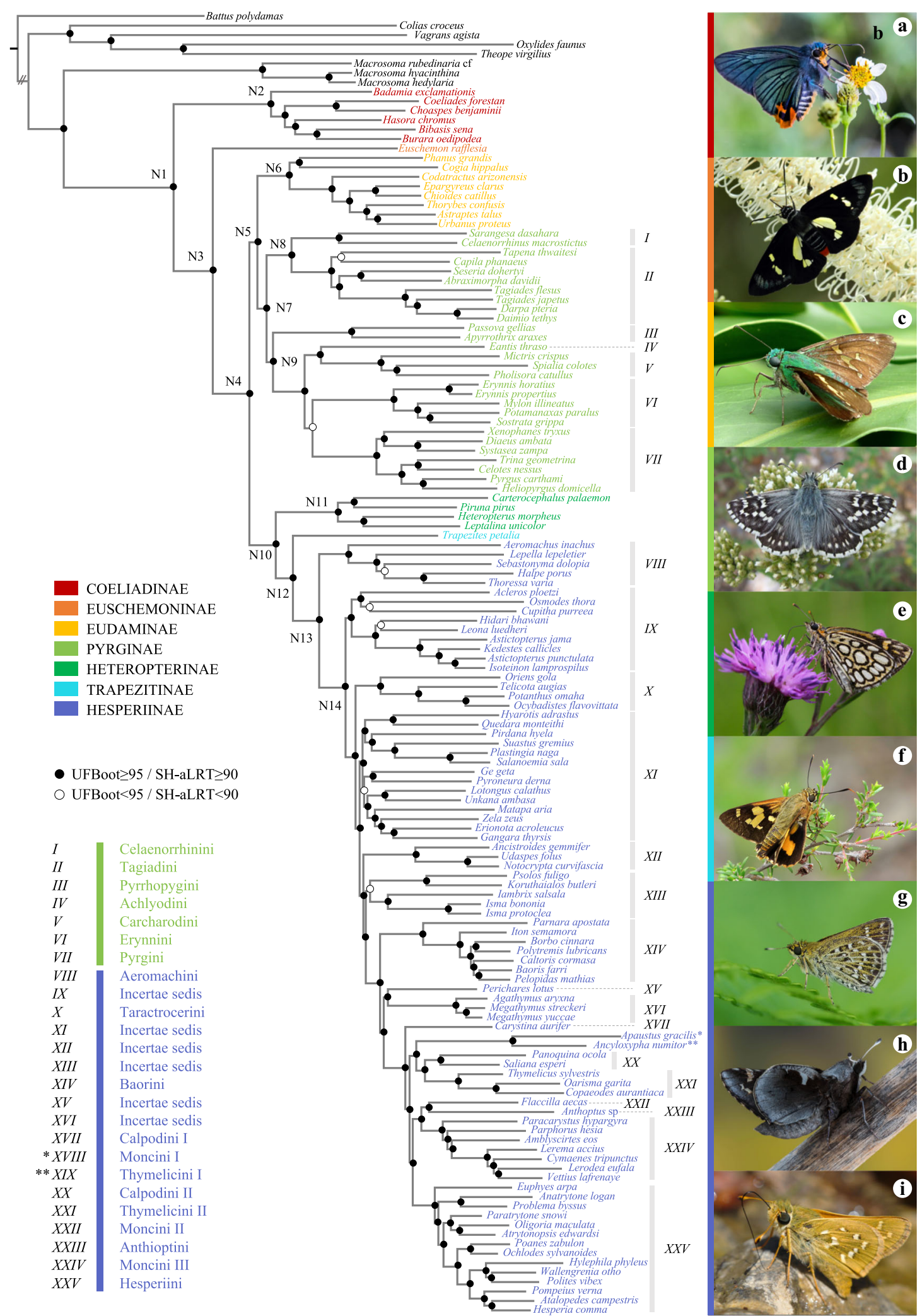

Fig. 2 (See legend on next page.) 
(See figure on previous page.)

Fig. 2 Phylogenomic skipper tree of life inferred using maximum likelihood. Maximum likelihood phylogeny inferred in IQ-TREE based on the nucleotide DT393 dataset with partitioning scheme selected in PartitionFinder and models of nucleotide substitution selected in IQ-TREE (Analysis A3). Nodal support values for numbered nodes on this tree (as well as alternative analyses) are presented in Fig. 3. Subfamilies and tribes recognized by Warren et al. [23] are indicated, and the color of species names indicates their subfamily. Images of skippers on the right illustrate morphological diversity within the family: a Choaspes benjaminii (credit: Sharleen Chao); b Euschemon rafflesia (credit: Todd Burrows); c Astraptes talus (credit: Les Catchick); d Pyrgus carthami (credit: Alan Cooper); e Heteropterus morpheus (credit: Hudák Tamás); f Trapezites symmomus (credit: John Tann); g Aeromachus inachus (credit: Tetsuya Shimizu); h Megathymus yuccae (credit: Jim \& Lynne Weber); i Hesperia comma (credit: Pedro Candela)

analyses except the amino acid ASTRAL-II analyses (A8), which resulted in low nodal support (ASTRALBoot $=51$, DT369; ASTRALBoot $=45$, DT393). All but one eudamine genus (Lobocla) is found in the Neotropics, and the two early lineages Coeliadinae (from Africa to Oceania) and Euschemoninae (Australia) are distributed in the Old World, a result with important biogeographical implications. An Old World tropical origin of skippers is suggested by the current distribution of Coeliadinae and Euschemoninae.

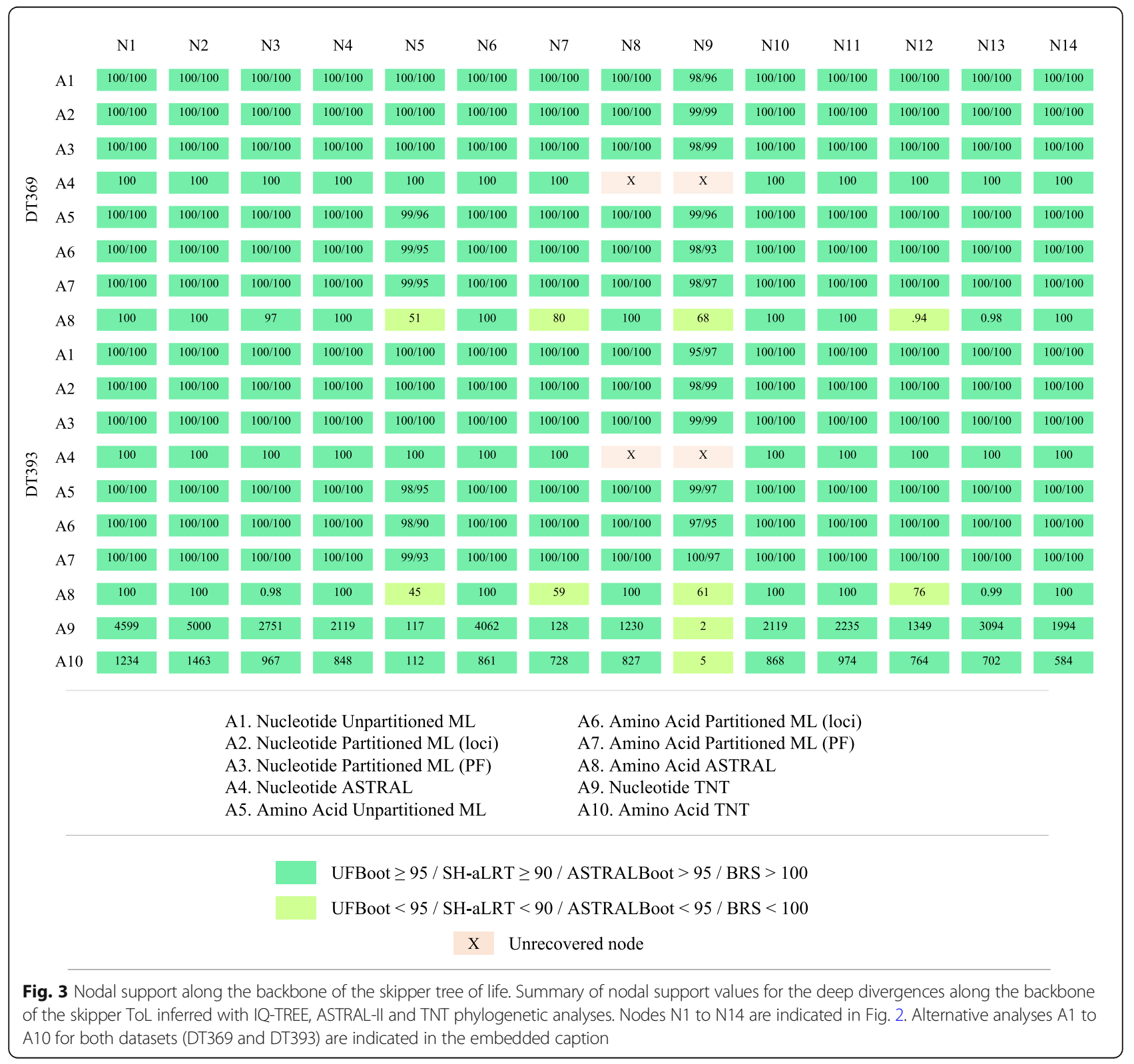


Our prediction of the Old World tropical origin of Hesperiidae is supported to some extent by the fossil record. The oldest known butterfly fossil is in the Coeliadinae and was discovered on the island of Fur in Denmark, embedded in marine deposits dating back to the Eocene (ca. $55 \mathrm{Ma}$ ), thereby substantiating the existence of skippers in the Old-World during that period [60], and supporting the colonization of the New World from the Old World. Multiple origins of New World and Old World skipper lineages are evident across our tree, indicating a dynamic biogeographical history over time. However, the true number of colonization events, mechanisms involved (dispersal, regional extinction, vicariance), and the biogeographic history of skippers will require additional research with greater taxon sampling.

Within Pyrginae, we recovered relationships different from those of prior phylogenetic studies. Celaenorrhinini (Clade I) and Tagiadini (Clade II) are sister groups, as in Warren et al. [23, 41], Sahoo et al. [32, 42] and Espeland et al. [21]. However, the placement of the Neotropical firetips, Pyrrhopygini (Clade III), remains unclear. Most analyses recovered the tribe as sister to all Pyrginae except Celaenorrhinini and Tagiadini (Fig. 2), but with little support as in [19]. The ASTRAL-II nucleotide analyses recovered an alternative topology for the placement of Pyrrhopygini, as the sister group to Celaenorrhinini and Tagiadini with moderate support $($ ASTRALBoot $=73$, DT369; ASTRALBoot $=85$, DT393) . This latter topological arrangement is consistent with results from previous studies [23, 32, 41, 42]. A greater and more diverse taxon sample could clarify the placement of firetips within Pyrginae. As in [19], we recovered Achlyodini (Clade IV) as sister to Carcharodini (Clade V), and Erynnini (Clade VI) as sister to Pyrgini (Clade VII), but with better support than in the previous study. These results are inconsistent with other previous studies that found alternative placements for these tribes within Pyrginae $[23,32,42]$.

Our results also point to Heteropterinae as the sister-group to Trapezitinae + Hesperiinae, a relationship that is robust and congruent with previous work [21, 23, 32, 41-43]. The Australasian subfamily Trapezitinae, represented here by a single species of Trapezites, is recovered as sister to the grass skippers, Hesperiinae (Figs. 1, 3). This sister-group relationship is robust across analyses and congruent with prior studies [21, 23, 32, 41, 42]. Within Hesperiinae, relationships among tribes and clades are not fully resolved. We find Aeromachini (Clade VIII) as sister to the rest of Hesperiinae with strong nodal support. Finally, the giant skippers (Agathymus and Megathymus spp., Clade XVI) were recovered as monophyletic with strong nodal support in all analyses (Figs. 2 and 3). Most inferences (IQ-TREE and ASTRAL-II) based on nucleotide datasets placed them as sister to the Neotropical genus Perichares, although amino acid datasets placed them as an isolated clade within Hesperiinae. Based on morphology and the species' known geographic distributions, the relationship of giant skippers with Perichares may be an artifact due to insufficient taxon sampling in this part of the tree and we do not consider this result to be robust. Several clades that remained unnamed by Warren et al. [23, 41] were also recovered in our analyses. We defer the proposal of new tribal names to a subsequent study with denser taxon sampling.

\section{Conclusions}

The current study provides a robust evolutionary framework that was largely lacking for one of the most species-rich butterfly families. Nearly all relationships in our tree are strongly supported, regardless of the dataset type (amino acids or nucleotides), optimality criterion (likelihood, parsimony, coalescence), or partitioning scheme. Our study demonstrates that AHE is a robust method for inferring phylogenies over a range of taxonomic levels using different optimality criteria. The unprecedented amount of data generated from this study will permit reconstruction of intricate evolutionary patterns across the skipper tree of life, and shed light on new ones.

\section{Additional files}

Additional file 1: Table of taxon sampling presenting detailed information for each specimen used in this study, along with number of captured AHE loci and genetic coverage in the AHE dataset. (XLSX $24 \mathrm{~kb}$ )

Additional file 2: Table presenting detailed information of the AHE matrix composition, including locus information, capture rate, probe region length, GC content and parsimony informative Sites. (XLSX 47 kb)

\section{Acknowledgements}

We thank Jesse Barber, Rod Eastwood, Harlan Gough, Dino Martins and Vladimir Lukhtanov for collecting specimens, and we are grateful to Samantha Epstein, Rachel Hawkins, Ryan St Laurent, and Luke Richards for assistance with specimen preparation, DNA extraction, and/or databasing. Chris Hamilton helped run several steps of the AHE pipeline. Vijay Barve helped check taxonomic data. We thank Todd Burrows, Pedro Candela, Les Catchick, Sharleen Chao, Alan Cooper, Tetsuya Shimizu, Hudák Tamás, Clement Tang, Jim Weber and Lynne Weber for their photographs of skippers. Motoki Saito helped develop some of the initial collaborative networks with specimen collectors, and Kiyoshi Maruyama assisted with skipper specimen identification. We acknowledge the University of Florida Research Computing for providing computational resources and support that contributed to the research results reported in this study.

\section{Funding}

A.Y.K., M.E, J.W.B, N.E.P., and D.J.L. acknowledge the US National Science Foundation (NSF) for financially supporting parts of this study (DEB-1541500, 1541557, 1541560). AVZB acknowledges support from a collaborative grant, Dimensions US-Biota-São Paulo: Assembly and evolution of the Amazon biota and its environment: an integrated approach, supported by the US National Science Foundation (DEB 1241056), National Aeronautics and Space Administration (NASA), and the Fundação de Amparo à Pesquisa do Estado de São Paulo (FAPESP Grant 2012/50260-6). 


\section{Availability of data and materials}

The datasets generated during the current study are available on Dryad (https://doi.org/10.5061/dryad.bq10q98).

\section{Authors' contributions}

EFAT and AYK conceived the study and supervised the molecular work: KD conducted the molecular work; EFAT performed the bioinformatic work with help from JWB and CE; EFAT performed the phylogenetic analyses, figure design, and led the writing with AYK; ADW, AVZB, AYK, JWB, MY, ME, NEP and DJL contributed data. All authors participated in drafting and revising the final manuscript. All authors read and approved the final manuscript.

\section{Ethics approval and consent to participate} Not applicable.

\section{Consent for publication}

Not applicable.

\section{Competing interests}

The authors declare that they have no competing interests.

\section{Publisher's Note}

Springer Nature remains neutral with regard to jurisdictional claims in published maps and institutional affiliations.

\section{Author details}

${ }^{1}$ Florida Museum of Natural History, University of Florida, Gainesville, Florida 32611, USA. ${ }^{2}$ RAPiD Genomics 747 SW 2nd Avenue IMB\#14, Gainesville, FL 32601, USA. ${ }^{3}$ Evolution and Ecology Group, Department of Biology, Middle Tennessee State University, Murfreesboro, TN 37132, USA. ${ }^{4}$ The University Museum, The University of Tokyo, Hongo, Bunkyo-ku, Tokyo 113-0033, Japan. ${ }^{5}$ Arthropoda Department, Zoological Research Museum Alexander Koenig, Adenauer Allee 160, 53113 Bonn, Germany. ${ }^{6}$ Department of Organismic and Evolutionary Biology, Harvard University, Cambridge, MA 02138, USA. ${ }^{7}$ Biology Department, City College of New York, City University of New York, New York, NY 10031, USA. ${ }^{8}$ Ph.D. Program in Biology, Graduate Center, City University of New York, New York, NY 10016, USA. 'Entomology Section, National Museum of the Philippines, 1000 Manila, Philippines.

\section{Received: 11 October 2017 Accepted: 7 June 2018}

Published online: 19 June 2018

\section{References}

1. Lemmon EM, Lemmon AR. High-throughput genomic data in systematics and phylogenetics. Annu Rev Ecol Evol Syst. 2013:44:99-121.

2. Jones MR, Good JM. Targeted capture in evolutionary and ecological genomics. Mol Ecol. 2016;25:185-202.

3. Lemmon AR, Emme SA, Lemmon EM. Anchored hybrid enrichment for massively high-throughput phylogenomics. Syst Biol. 2012;61:727-44.

4. St Laurent, R.A., Hamilton, C.A. and Kawahara, A.Y. Museum specimens provide phylogenomic data to resolve relationships of sack-bearer moths (Lepidoptera, Mimallonoidea, Mimallonidae). Syst. Entomol. 2018. https://doi. org/10.1111/syen.12301.

5. Prum RO, Berv JS, Dornburg A, Field DJ, Townsend JP, Moriarty Lemmon E, et al. A comprehensive phylogeny of birds (Aves) using targeted nextgeneration DNA sequencing. Nature. 2015;526:569-73.

6. Hackett SJ, Kimball RT, Reddy S, Bowie RCK, Braun EL, Braun MJ, et al. A phylogenomic study of birds reveals their evolutionary history. Science. 2008;320:1763-8.

7. Tucker DB, Colli GR, Giugliano LG, Hedges SB, Hendry CR, Lemmon EM, et al. Methodological congruence in phylogenomic analyses with morphological support for teiid lizards (Sauria: Teiidae). Mol. Phylogenet. Evol. 2016;103:75-84.

8. Stout CC, Tan M, Lemmon AR, Lemmon EM, Armbruster JW. Resolving Cypriniformes relationships using an anchored enrichment approach. BMC Evol Biol. 2016;16:244.

9. Maddison WP, Evans SC, Hamilton CA, Bond JE, Lemmon AR. Lemmon EM A genome-wide phylogeny of jumping spiders (Araneae, Salticidae), using anchored hybrid enrichment. Zookeys. 2017;695:89-101.
10. Fragoso-Martínez I, Salazar GA, Martínez-Gordillo M, Magallón S, SánchezReyes L, Moriarty Lemmon E, et al. A pilot study applying the plant anchored hybrid enrichment method to new world sages (Salvia subgenus Calosphace ; Lamiaceae). Mol. Phylogenet. Evol. 2017;117:124-34.

11. Mitchell N, Lewis PO, Lemmon EM, Lemmon AR, Holsinger KE. Anchored phylogenomics improves the resolution of evolutionary relationships in the rapid radiation of Protea L. Am J Bot. 2017;104:102-15.

12. Domingos FMCB, Colli GR, Lemmon A, Lemmon EM, Beheregaray LB. In the shadows: phylogenomics and coalescent species delimitation unveil cryptic diversity in a Cerrado endemic lizard (Squamata: Tropidurus). Mol Phylogenet Evol. 2017;107:455-65.

13. Ruane S, Raxworthy CJ, Lemmon AR, Lemmon EM, Burbrink FT. Comparing species tree estimation with large anchored phylogenomic and small sanger-sequenced molecular datasets: an empirical study on Malagasy pseudoxyrhophiine snakes. BMC Evol Biol. 2015;15:221.

14. Hamilton CA, Lemmon AR, Lemmon EM, Bond JE. Expanding anchored hybrid enrichment to resolve both deep and shallow relationships within the spider tree of life. BMC Evol Biol. 2016;16:212.

15. Brandley MC, Bragg JG, Singhal S, Chapple DG, Jennings CK, Lemmon AR, et al. Evaluating the performance of anchored hybrid enrichment at the tips of the tree of life: a phylogenetic analysis of Australian Eugongylus group scincid lizards. BMC Evol Biol. 2015;15:62.

16. Young $A D$, Lemmon AR, Skevington JH, Mengual $X$, Ståhls $G$, Reemer $M$, et al. Anchored enrichment dataset for true flies (order Diptera) reveals insights into the phylogeny of flower flies (family Syrphidae). BMC Evol Biol. 2016;16:143.

17. Winterton SL, Lemmon AR, Gillung JP, Garzon IJ, Badano D, Bakkes DK, et al. Evolution of lacewings and allied orders using anchored phylogenomics (Neuroptera, Megaloptera, Raphidioptera). Syst Entomol. 2018;43:330-54.

18. Haddad S, Shin S, Lemmon AR, Lemmon EM, Svacha P, Farrell BD, et al. Anchored hybrid enrichment provides new insights into the phylogeny and evolution of longhorned beetles (Cerambycidae). Syst Entomol. 2018;43:6889.

19. Shin S, Clarke DJ, Lemmon AR, Lemmon EM, Alexander L, Haddad S, et al. Phylogenomic data yield new and robust insights into the phylogeny and evolution of weevils. Mol Biol Evol. 2018;35:823-36.

20. Breinholt JW, Earl C, Lemmon AR, Lemmon EM, Xiao L, Kawahara AY. Resolving relationships among the megadiverse butterflies and moths with a novel pipeline for anchored phylogenomics. Syst Biol. 2018;67: 78-93.

21. Espeland M, Breinholt J, Willmott KR, Warren AD, Vila R, Toussaint EFA, et al. A comprehensive and dated Phylogenomic analysis of butterflies. Curr Biol. 2018;28:770-8.

22. Van Nieukerken EJ, Kaila L, Kitching IJ, Kristensen NP, Lees DC, Minet J, et al. Order Lepidoptera Linnaeus, 1758. In: Zhang Z-Q, editor. Anim. Biodivers. An Outl. Higher-level Classif. Surv. Taxon. Richness. Auckland: Magnolia Press; 2011. p. 212-21.

23. Warren AD, Ogawa JR, Brower AVZ. Revised classification of the family Hesperiidae (Lepidoptera: Hesperioidea) based on combined molecular and morphological data. Syst Entomol. 2009;34:467-523.

24. Bauder JAS, Warren AD, Krenn HW. The ecological role of extremely longproboscid Neotropical butterflies (Lepidoptera: Hesperiidae) in plantpollinator networks. Arthropod Plant Interact. 2015;9:415-24.

25. Broady-Venables BA, Barrows EM. Skippers: pollinators or nectar thieves? J Lepid Soc. 1985;39:299-312.

26. Suetsugu K, Tanaka K. Diurnal butterfly pollination in the orchid Habenaria radiata. Entomol Sci. 2014;17:443-5.

27. Goulson D, Ollerton J, Sluman C. Foraging strategies in the small skipper butterfly,Thymelicus flavus: when to switch? Anim Behav. 1997;53:1009-16.

28. Primack R, Howe $H$. Interference competition between a hummingbird (Amazilia tzacat) and skipper butterflies (Hesperiidae). Biotropica. 1975;7:55-8.

29. Thomas JA, Thomas CD, Simcox DJ, Clarke RT. Ecology and declining status of the silver-spotted skipper butterfly (Hesperia comma) in Britain. J Appl Ecol. 1986;23:365-80.

30. Davies ZG, Wilson RJ, Brereton TM, Thomas CD. The re-expansion and improving status of the silver-spotted skipper butterfly (Hesperia comma) in Britain: a metapopulation success story. Biol Conserv. 2005;124:189-98.

31. Mau RF, Murai K, Kumashiro B, Teramoto K. Biological control of the banana skipper, Pelopidas thrax (Linnaeus), (Lepidoptera: Hesperiidae) in Hawaii. Proc Hawaiian Entomol Soc. 1980;23:231-8. 
32. Sahoo RK, Warren AD, Collins SC, Kodandaramaiah U. Hostplant change and paleoclimatic events explain diversification shifts in skipper butterflies (family: Hesperiidae). BMC Evol Biol. 2017;17:174.

33. Wahlberg $\mathrm{N}$, Wheat $\mathrm{CW}$, Peña $\mathrm{C}$. Timing and patterns in the taxonomic diversification of Lepidoptera (butterflies and moths). PLoS One. 2013;8:1-8.

34. Haase E. Zum System der Tagfalter. Dtsch. Entomol. Zeitschrift "Iris". 1891:4:1-33.

35. Latreille PA. Genera Crustaceorum et Insectorum secundum ordinem naturalem in familias disposita, iconibus exemplisque plurimis explicata. Paris: Parislis et Argentorati, Armand König. p. 1809

36. Mabille MP. Catalogue des Hespérides du Musée Royal d'Histoire Naturelle de Bruxelles. Ann la Société Entomol Belgique. 1878;21:12-44.

37. Weller SJ, Pashley DP. In search of butterfly origins. Mol Phylogenet Evol. 1995;4:235-46.

38. Heikkilä M, Kaila L, Mutanen M, Peña C, Wahlberg N. Cretaceous origin and repeated tertiary diversification of the redefined butterflies. Proc R Soc B Biol Sci. 2012;279:1093-9.

39. Kawahara AY, Breinholt JW, Espeland M, Storer CG, Plotkin D, Dexter KM et al. Phylogenetics of moth-like butterflies (Papilionoidea: Hedylidae) based on a new 13-locus target capture probe set. Mol Phylogenet Evol. 2018. https://doi.org/10.1016/j.ympev.2018.06.002.

40. Kawahara AY, Breinholt JW. Phylogenomics provides strong evidence for relationships of butterflies and moths. Proc R Soc B Biol Sci. 2014;281: 20140970.

41. Warren AD, Ogawa JR, Brower AVZ. Phylogenetic relationships of subfamilies and circumscription of tribes in the family Hesperiidae (Lepidoptera: Hesperioidea). Cladistics. 2008;24:642-76.

42. Sahoo RK, Warren AD, Wahlberg N, Brower AVZ, Lukhtanov VA, Kodandaramaiah $U$. Ten genes and two topologies: an exploration of higher relationships in skipper butterflies (Hesperiidae). PeerJ. 2016;4:e2653.

43. Zhang J, Cong Q, Fan X-L, Wang R, Wang M, Grishin NV. Mitogenomes of giant-skipper butterflies reveal an ancient split between deep and shallow root feeders. F1000Research. 2017:6:222.

44. Cho S, Epstein SW, Mitter K, Hamilton CA, Plotkin D, Mitter C, et al. Preserving and vouchering butterflies and moths for large-scale museumbased molecular research. PeerJ. 2016;4:e2160.

45. Edgar RC. Search and clustering orders of magnitude faster than BLAST. Bioinformatics. 2010;26:2460-1.

46. Chang Z, Li G, Liu J, Zhang Y, Ashby C, Liu D, et al. Bridger: a new framework for de novo transcriptome assembly using RNA-seq data. Genome Biol. 2015:16:30

47. Camacho C, Coulouris G, Avagyan V, Ma N, Papadopoulos J, Bealer K, et al. BLAST+: architecture and applications. BMC Bioinformatics. 2009;10:421

48. Zhan S, Merlin C, Boore JL, Reppert SM. The monarch butterfly genome yields insights into long-distance migration. Cell. 2011;147:1171-85.

49. Katoh K, Standley DM. MAFFT multiple sequence alignment software version 7: improvements in performance and usability. Mol. Biol. Evol. 2013; 30:772-80.

50. Kück P, Longo GC. FASconCAT-G: extensive functions for multiple sequence alignment preparations concerning phylogenetic studies. Front Zool. 2014;11:81.

51. Larsson A. AliView: a fast and lightweight alignment viewer and editor for large datasets. Bioinformatics. 2014;30:3276-8.

52. Nguyen LT, Schmidt HA, Von Haeseler A, Minh BQ. IQ-TREE: a fast and effective stochastic algorithm for estimating maximum-likelihood phylogenies. Mol. Biol. Evol. 2015;32:268-74.

53. Lanfear R, Frandsen PB, Wright AM, Senfeld T, Calcott B. Partitionfinde 2: new methods for selecting partitioned models of evolution for molecular and morphological phylogenetic analyses. Mol Biol Evol. 2017;34:772-3.

54. Soubrier J, Steel M, Lee MSY, Der Sarkissian C, Guindon S, Ho SYW, et al. The influence of rate heterogeneity among sites on the time dependence of molecular rates. Mol. Biol. Evol. 2012;29:3345-58.

55. Minh $B Q$, Nguyen MAT, Von Haeseler A. Ultrafast approximation for phylogenetic bootstrap. Mol. Biol. Evol. 2013;30:1188-95.

56. Guindon S, Dufayard JF, Lefort V, Anisimova M, Hordijk W, Gascuel O. New algorithms and methods to estimate maximum-likelihood phylogenies: assessing the performance of PhyML 3.0. Syst Biol. 2010;59:307-21.
57. Mirarab S, Warnow T. ASTRAL-Il: coalescent-based species tree estimation with many hundreds of taxa and thousands of genes. Bioinformatics. 2015;31:144-52.

58. Goloboff PA, Catalano SA. TNT version 1.5, including a full implementation of phylogenetic morphometrics. Cladistics. 2016:32:221-38.

59. O'Neil ST, Dzurisin JD, Carmichael RD, Lobo NF, Emrich SJ, Hellmann JJ. Population-level transcriptome sequencing of nonmodel organisms Erynnis propertius and Papilio zelicaon. BMC Genomics. 2010;11:310.

60. de Jong R. Reconstructing a 55-million-year-old butterfly (Lepidoptera: Hesperiidae). Eur J Entomol. 2016;113:423-8.

\section{Ready to submit your research? Choose BMC and benefit from:}

- fast, convenient online submission

- thorough peer review by experienced researchers in your field

- rapid publication on acceptance

- support for research data, including large and complex data types

- gold Open Access which fosters wider collaboration and increased citations

- maximum visibility for your research: over $100 \mathrm{M}$ website views per year

At BMC, research is always in progress.

Learn more biomedcentral.com/submissions 